\title{
Absence of the liquid-liquid phase transition in aqueous ionic liquids
}

\author{
Johannes Bachler (®), Lilli-Ruth Fidler, and Thomas Loerting (๑* \\ Institute of Physical Chemistry, University of Innsbruck, Innrain 52c, A-6020 Innsbruck, Austria
}

(Received 6 October 2020; accepted 18 November 2020; published 11 December 2020)

\begin{abstract}
The anomalies of supercooled water may be explained by an underlying liquid-liquid phase transition (LLPT) between high- and low-density states. Recently, its observation at $185 \mathrm{~K}$ was inferred using solutions containing aqueous ionic liquids at a solute mole fraction of $x=0.156$ [Woutersen et al., Science 359, 1127 (2018)]. We employ x-ray diffraction, calorimetry, and dilatometry on these hydrazinium trifluoroacetate solutions at $x=0.00-0.40$ to show that the transition at $185 \mathrm{~K}$ is not related to a genuine LLPT of water. Continuous densification upon compression, continuous changes of halo position, and absence of thermal signatures for a high- to low-density transition rule out the possibility of an LLPT for $x \geqslant 0.13$. The data show that employing sophisticated solutions adds a layer of complexity that hampers extrapolation of the LLPT concept from oneto two-component systems. The possibility of an LLPT can only be probed for pure water or sufficiently dilute aqueous solutions.
\end{abstract}

DOI: 10.1103/PhysRevE.102.060601

Water is ubiquitous, encountered all over the Earth and in nearly every corner of our universe. Its liquid state is the perfect host for all kinds of solutes ranging from salts and simple molecular compounds to complex biomolecules and polymers. Despite its simple chemical constitution, water is far from trivial and hardly understood. This alone is impressively illustrated by the list of its anomalies counting over 70 entries [1]. Among these, the unusual, diverging behavior of thermodynamic response functions (such as isobaric heat capacity) upon supercooling has puzzled scientists for decades [2]. In this context, the liquid-liquid critical point (LLCP) scenario has gained particular interest in recent years [3]. The scenario involves a second critical point around 220 $\mathrm{K}$ below which water separates into two liquids: low-density liquid (LDL) and high-density liquid (HDL). At low temperatures, these liquids are encountered in their glassy states, low-density amorphous (LDA) and high-density amorphous (HDA) ice [4]. However, evidence pointing toward this in real water is still discussed controversially [5]. This is mainly because the observation of the liquid-liquid phase transition (LLPT) emanating from the purported LLCP is hampered by rapid ice crystallization in the so-called "no man's land" at $150 \mathrm{~K}<T<238 \mathrm{~K}$. To learn more about liquid water in the no man's land, scientists have resorted to ultrafast methods, both in simulations [6] and experiments [7] to avoid crystallization. Yet another method is to delay ice formation by adding solutes. Binary solutions of salts (mostly $\mathrm{LiCl}$ ) and alcohols (mostly glycerol) have been studied intensely [8-10].

\footnotetext{
*thomas.loerting@uibk.ac.at
}

Published by the American Physical Society under the terms of the Creative Commons Attribution 4.0 International license. Further distribution of this work must maintain attribution to the author(s) and the published article's title, journal citation, and DOI.
Here, we find ourselves caught in a fundamental dilemma: If the concentration is too low the solution still crystallizes; if it is too high the anomalies are suppressed and no information on water is gained. More specifically, adding solutes such as salts or glycerol gradually suppresses water's anomalies and forces the LLPT to lower pressures and temperatures [10]. As quite large amounts of solute have to be added to avoid crystallization in the no man's land, these solutions also display completely suppressed anomalies and no LLPT at positive pressures. That is, the use of concentrated solutions is disqualified if the aim is to search for a LLPT relating to pure water at $150 \mathrm{~K}<T<238 \mathrm{~K}$ [11].

Recently, Zhao and Angell [12] and Woutersen et al. [13] seem to have found a way around this decades-old dilemma. They claim that the ionic liquid hydrazinium trifluoroacetate $\left(\mathrm{N}_{2} \mathrm{H}_{5}{ }^{+} \mathrm{TFA}^{-}\right)$mixes ideally with water and effectively eliminates the no man's land by suppressing crystallization, yet still featuring the heat capacity and density anomalies expected for pure water. From the thermodynamic point of view, ideal mixing necessitates a mixing enthalpy of zero, which is manifested in identical water-solute, water-water, and solutesolute interactions. In other words, the solute is said to fit perfectly into the H-bond network of water without perturbing it. They provide infrared (IR) spectroscopy and molecular dynamics simulations to make the case for hydrogen bonding very similar to pure water. Only water molecules in the second hydration shell appear to be more disordered and to resemble pressurized liquid water. This resonates well with the notion that salt content $x$ and external pressure $p$ have similar effects on the structure of water [14]. Based on these assumptions, they believe the solution at $x=0.156$ to be at an effective pressure of $0.15-0.20 \mathrm{GPa}$, at which the LLPT from HDL to LDL can be realized simply by cooling. Their key evidence to argue for this scenario is the observation of an exothermic first-order transition near $185 \mathrm{~K}$ upon cooling. Due to similarities of the IR spectra with pure HDA and LDA 

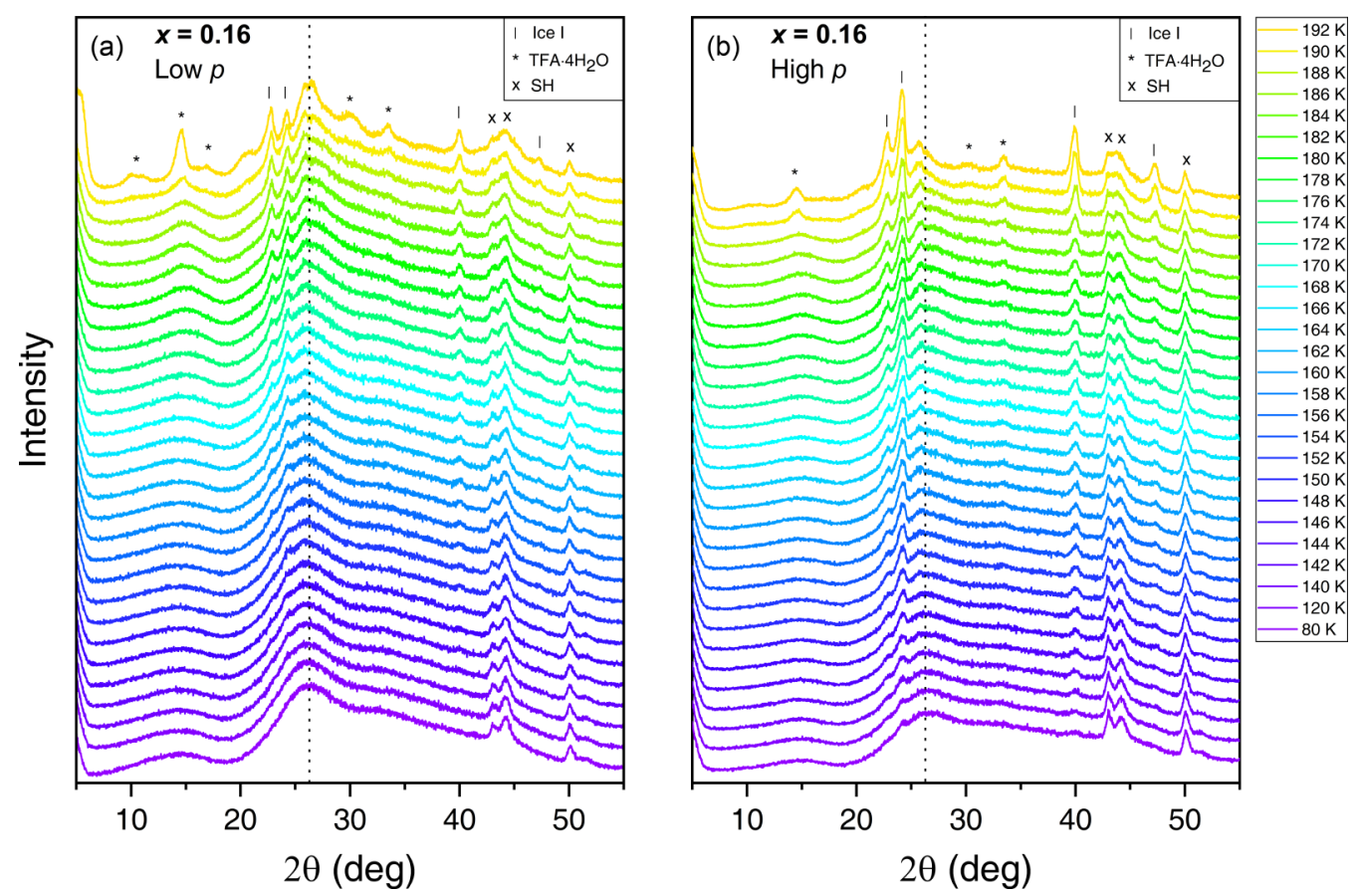

FIG. 1. Selected $\mathrm{x}$-ray diffraction patterns of a vitrified aqueous $\mathrm{N}_{2} \mathrm{H}_{5}{ }^{+} \mathrm{TFA}^{-}$solution $(x=0.16)$ upon heating from 80 to $192 \mathrm{~K}$, (a) Before high-pressure treatment and (b) after pressurization to $1.8 \mathrm{GPa}$ at $80 \mathrm{~K}$. The dotted line represents the halo peak maximum of the most intense halo peak at $26.3^{\circ}$. Bragg peak positions of ice I, TFA $\cdot 4 \mathrm{H}_{2} \mathrm{O}$ and the sample holder $(\mathrm{SH})$ are marked by ticks, asterisks, and crosses, respectively. Scans in the full temperature range and the assignment of Bragg peaks are shown in the Supplemental Material [15].

spectra, this transition was attributed to an LLPT from HDL to LDL, which then vitrifies to LDA upon further cooling. Reheating reverts the transition sequence: LDA first experiences the glass-to-liquid transition to LDL, which appears to transform back to HDL via an endothermic event at $190 \mathrm{~K}$ $[12,13]$. Only at higher temperatures, HDL crystallizes to ice. This interpretation is schematically illustrated in Fig. S1 of the Supplemental Material [15]. However, whether this LLPT truly involves high-density and low-density water still demands more evidence. Mole fractions of $x=0.16$ correspond to roughly $1.5 \mathrm{~kg}$ of ionic liquid per $\mathrm{kg}$ water, an amount too excessive to take waterlike behavior for granted. Therefore, detailed studies on the properties of such solutions using methods other than IR spectroscopy are particularly desirable. Another missing key experiment is the crossing of the phase boundary between low- and high-density water by compression of the low-density amorphous state.

In this work we provide a combination of x-ray diffraction, high-pressure experiments and calorimetry experiments to demonstrate that a transition between two liquids (or glasses), without compositional changes, does not, in fact, take place. Aqueous $\mathrm{N}_{2} \mathrm{H}_{5}{ }^{+} \mathrm{TFA}^{-}$solutions cannot be treated like pure water, and accordingly scenarios for water are invalid to describe the phase behavior of solutions at $x=0.16$. However, by decreasing the solute concentration, waterlike behavior is restored, including crystallization in the no man's land.

$X$-ray diffraction: Solution structure. X-ray diffraction is a powerful tool for probing the atomistic structure not only of crystals, but also of liquids and glasses. In pure water, HDA and LDA are distinguished by their broad halo peaks at distinct angles marking the positions of oxygen atoms in the amorphous solid [16]. The peak maxima are found at $2 \theta=$ $24.0^{\circ}$ and $2 \theta=28.5^{\circ}\left(\mathrm{Cu} K \alpha_{1}\right)$ for LDA and HDA, respectively. Figure 1(a) shows X-ray diffractograms of the vitrified solution with $x=0.16$ at $80 \mathrm{~K}$. At $80 \mathrm{~K}$, the solution is indeed amorphous as indicated by the absence of Bragg peaks. A broad and intense halo peak is predominant at $2 \theta=26.3^{\circ}$, accompanied by a less intense halo around $2 \theta=14.5^{\circ}$ and a broad shoulder at $2 \theta \approx 30^{\circ}-40^{\circ}$. The most striking difference to the diffractograms of pure LDA or HDA is the presence of additional halo peaks. The intense halo located at $2 \theta=26.3^{\circ}$ could correspond to LDA-like water where the upshift from $2 \theta=24.0^{\circ}$ is due to the presence of the ionic liquid as solute. The broad shoulder might indicate some more closely packed water molecules, similar to HDA or VHDA [17]. The halo at $2 \theta=14.5^{\circ}$ is of unknown nature. Broad shoulders and even double halo peaks, which also emerge in the liquid state at room temperature (see Fig. S2 in the Supplemental Material [15]), are the first signs that we are not dealing with an ideal, waterlike solution. Non-ideality can also be inferred based on the acidity of hydrazinium, where the $\mathrm{pH}$ of the $x=0.16$ solution is 3.7 [15]. While dissociation of hydrazinium is not taken into account in the work of Ref. [13], it is crucial to consider because an excess of protons perturbs the hydrogen bond network and causes nonideal mixing.

$X$-ray diffraction: Polyamorphic behavior at low pressure? The established criteria for polyamorphic behavior for pure water involve a sharp transition to LDA upon heating pure HDA, which is manifested as a jumplike change in halo maximum position from $2 \theta=28.5^{\circ}$ to $2 \theta=24.0^{\circ}$ [18]. Nothing similar is seen upon cooling and heating through the transition at $185 \mathrm{~K}$ for the $\mathrm{N}_{2} \mathrm{H}_{5}{ }^{+} \mathrm{TFA}^{-}$solution. In Fig. 1(a) we show selected diffractograms upon reheating from 80 to $192 \mathrm{~K}$. The claim by Woutersen et al. [13] that a sharp transition 

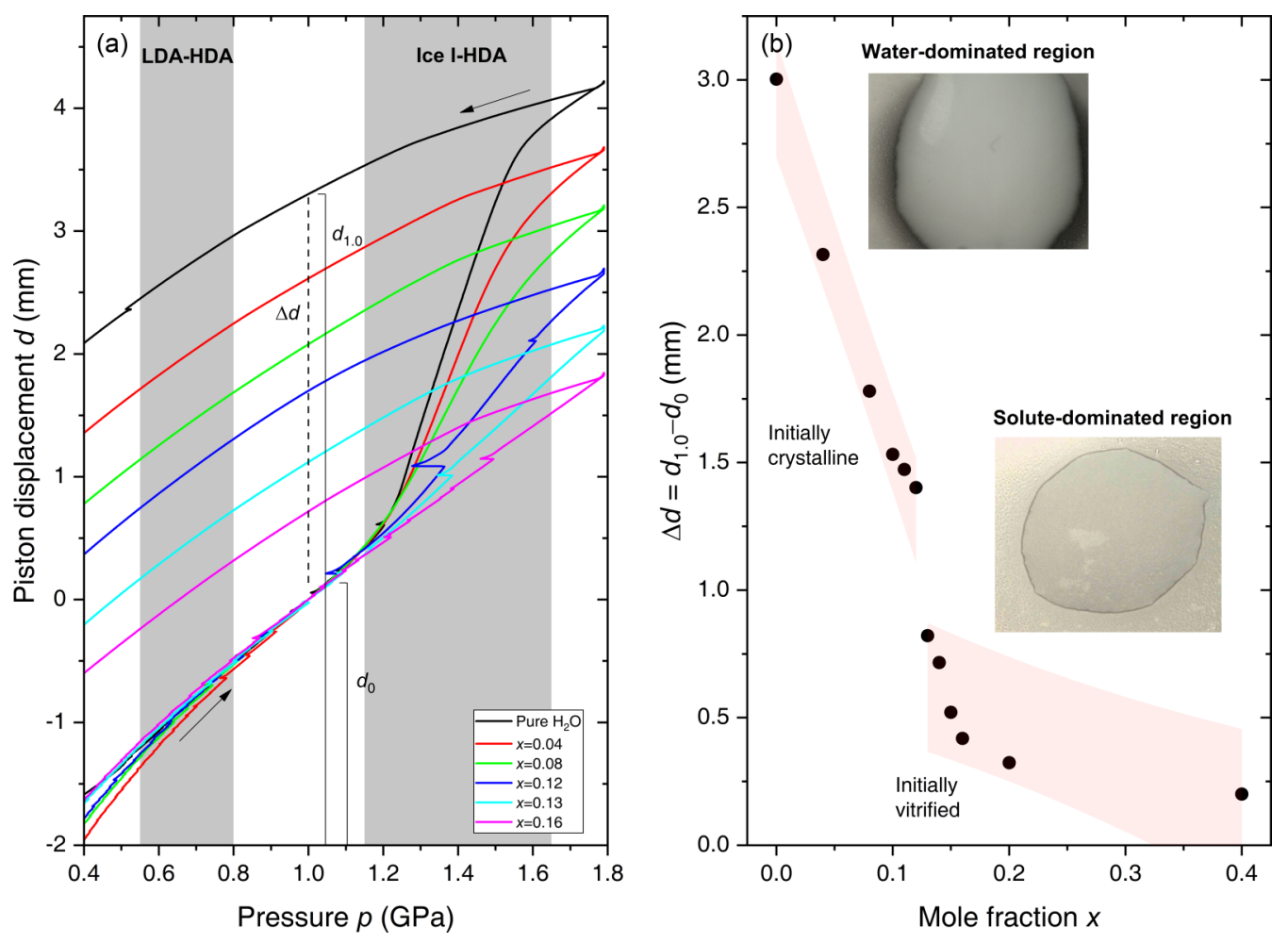

FIG. 2. (a) Piston displacement (indicating density changes) during compression and decompression of selected quenched $\mathrm{N}_{2} \mathrm{H}_{5}{ }^{+} \mathrm{TFA}{ }^{-}-$ $\mathrm{H}_{2} \mathrm{O}$ solutions at $77 \mathrm{~K}$ (mole fractions as indicated). Curves are aligned at $d=0$ and $1.0 \mathrm{GPa}$ for better comparison. Gray areas indicate the LDA $\rightarrow$ HDA and ice I $\rightarrow$ HDA transition in pure water. The zigzagging of some curves upon compression indicates small pressure drops caused by friction of the moving piston [20]. (b) Permanent densification $\Delta d=d_{1.0}-d_{0}$ between up- and downstroke at 1.0 GPa of all studied solutions [as defined by the dashed line in Fig. 2(a)]. The broad red bands highlight the switch from vitrified to crystallized samples between $x=0.12$ and 0.13 . The insets show optical-cryomicroscopy images for $x=0.16$ (bottom) and $x=0.04$ (top). Compressed samples are recovered at $77 \mathrm{~K}$ and used for subsequent characterization.

from low-density water to high-density water occurs at $190 \mathrm{~K}$ necessitates a halo peak maximum that abruptly shifts by $\approx 4.5^{\circ}$. However, the peak maximum remains at $2 \theta=26.3^{\circ}$ [dotted line in Fig. 1(a)] throughout the entire observed temperature range. Consequently, the purported LLPT related to polyamorphism in pure water is not confirmed by our diffraction data here. Even crystallization of ice I [marked by ticks in Fig. 1(a)] that sets in right after the ambiguous transition or crystallization of TFA $\cdot 4 \mathrm{H}_{2} \mathrm{O}$ hydrate beginning at $\approx 192 \mathrm{~K}$ [15] has no influence on the halo position.

Dilatometry and $x$-ray diffraction: Polyamorphic behavior at high pressure? Halo peak positions and their temperature dependence are not the only means to identify behavior similar to that of pure water. In pure bulk water, LDA exhibits a transformation to HDA at $\approx 0.6 \mathrm{GPa} / 77 \mathrm{~K}$ involving a sudden, steplike densification of roughly $25 \%$ (see Fig. 2 in Ref. [19]). If the $\mathrm{N}_{2} \mathrm{H}_{5}{ }^{+} \mathrm{TFA}^{-}$solution were to behave like pure water pressurized to $0.15-0.20 \mathrm{GPa}$ it would also need to exhibit the sharp LDA $\rightarrow$ HDA transformation upon further pressurization at $77 \mathrm{~K}$ at $\approx 0.4 \mathrm{GPa}$. The volume change of selected aqueous $\mathrm{N}_{2} \mathrm{H}_{5}{ }^{+} \mathrm{TFA}^{-}$solutions during compression up to $1.8 \mathrm{GPa}$ is presented in Fig. 2(a) for several mole fractions. For $x=0.16$ (pink line) there is no sign of a step and hence, no abrupt volume change.

Interestingly, a steplike transition appears when decreasing the mole fraction from $x=0.16$ to $x=0.12$ [see Fig. 2(a), blue line]. Its onset pressure of $1.1 \mathrm{GPa}$ is different from the onset pressure of $<0.6 \mathrm{GPa}$ expected for the LDA $\rightarrow$ HDA transition. Instead, the onset exactly matches the onset pressure for pressure-induced amorphization of pure hexagonal ice at $77 \mathrm{~K}$, yielding an HDA state [21] [see gray bar in Fig. 2(a)]. That is, for the $x=0.12$ solution the contribution of $\mathrm{N}_{2} \mathrm{H}_{5}{ }^{+}$TFA $^{-}$is small enough to enable crystallization of ice upon cooling. Only the ice fraction experiences the transition to HDA, but no LDA. The amount of ice after cooling at 1 bar increases when decreasing $x$, up to the point where the sample crystallizes fully to ice I at $x=0$. Consequently, pure water [black line in Fig. 2(a)] experiences the full 25\% density change at the ice I $\rightarrow$ HDA transition, which corresponds to a piston displacement $\Delta d$ of $3.0 \mathrm{~mm}$. $\Delta d$ is evaluated at 1.0 GPa [see Fig. 2(a), vertical dashed line] and plotted for all solutions in Fig. 2(b). Starting from pure water, the permanent densification $\Delta d$ indicated in Fig. 2(b) first decreases rather linearly with $x$, i.e., less and less ice is available for pressure amorphization. Between $x=0.12$ and $x=0.13$ $\Delta d$ suddenly drops from 1.4 to $0.8 \mathrm{~mm}$. This sudden and large decline indicates that the nature of the sample changes fundamentally. Ice no longer precipitates at $x \geqslant 0.13$, but the solution vitrifies homogeneously. This is also evidenced in the optical microscopy images in Fig. 2(b), where the images change from opaque to transparent at $x \geqslant 0.13$. Opacity indicates that parts of the sample have crystallized, whereas transparency indicates glassy nature. In other words, crystallization in the no man's land is only avoided for $x \geqslant 0.13$ but 


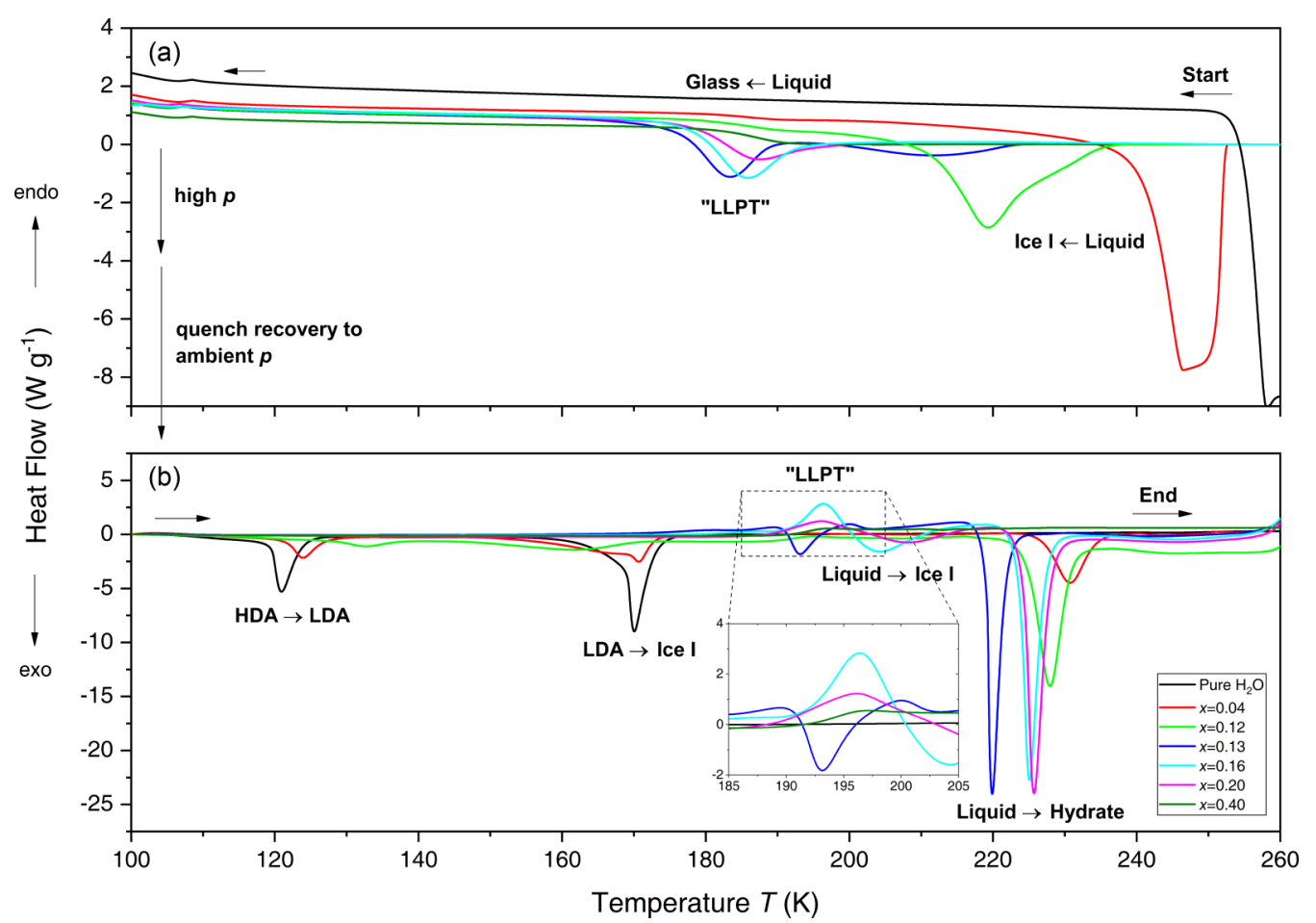

FIG. 3. DSC traces $\left(30 \mathrm{~K} / \mathrm{min}\right.$ ) of $\mathrm{N}_{2} \mathrm{H}_{5}{ }^{+} \mathrm{TFA}^{-}$-water solutions at the indicated mole fractions $x$. (a) Cooling scans at ambient pressure and (b) heating scans of recovered samples after pressurization to $1.8 \mathrm{GPa}$ at $80 \mathrm{~K}$. Curves are normalized by weight of solution and aligned. The inset shows a magnification of selected samples near $185 \mathrm{~K}$. Scans covering more compositions in this concentration range are displayed in Figs. S5 (water-dominated region) and S6 (solute-dominated region) of the Supplemental Material [15].

waterlike crystallization is encountered for less concentrated solutions, thereby restoring the dilemma mentioned above. This sudden switch from water-dominated behavior at $x<$ 0.13 to solute-dominated behavior at $x \geqslant 0.13$ is consistent with similar findings for $\mathrm{LiCl}-\mathrm{H}_{2} \mathrm{O}[22,23]$ and glycerol- $\mathrm{H}_{2} \mathrm{O}$ [24].

$\Delta d$ in the solute-dominated regime is still non zero, and so it is of interest to check for the origin of the densification, especially if there are signs for densified amorphous material (HDA-like) in spite of the absence of jumplike changes. Figure 1(b) shows the x-ray patterns after the pressurizationdepressurization cycle shown in Fig. 2(a). However, these patterns are indistinguishable from the patterns before pressurization. Also, upon heating, barely any differences can be noted between patterns before and after pressurization. In other words, no signs of polyamorphism are observed for the $\mathrm{N}_{2} \mathrm{H}_{5}{ }^{+} \mathrm{TFA}^{-}$solution-in stark contrast to the situation in pure water.

Differential scanning calorimetry: Complementing the picture. The arguments presented so far against polyamorphism in concentrated $\mathrm{N}_{2} \mathrm{H}_{5}{ }^{+} \mathrm{TFA}^{-}$solutions are further reinforced by our differential scanning calorimetry (DSC) data. Figure 3(a) depicts selected cooling scans at ambient pressure, without any pressurization involved. Pure water and solutions up to $x=0.12$ first show a massive exotherm. This signals precipitation of ice I, as has been inferred above from densitypressure curves in Fig. 2(a) and the microscopy images in Fig. 2(b). Freezing of ice for $x \leqslant 0.12$ causes the solute concentration in the remaining solution to rise. Upon continued cooling the freeze-concentrated solution (FCS) experiences a liquid-to-glass transition, i.e., it immobilizes. The change in heat capacity associated with the loss of the translational degree of freedom is seen as a subtle step change in the heat flow at $T_{g} \approx 185 \mathrm{~K}$. Such thermal behavior is characteristic for water-rich solutions containing glass formers, e.g., $\mathrm{LiCl}$ [22], glycerol [25,26], and sucrose [27]. Thus, it is reasonable to presume that also $\mathrm{N}_{2} \mathrm{H}_{5}{ }^{+} \mathrm{TFA}^{-}$mixtures of $x \leqslant 0.12$ are composed of ice and patches of glassy FCS below $T_{g}$. With increasing mole fraction, the ratio of ice to FCS decreases, leading to the decrease in permanent densification as demonstrated in Fig. 2(b). Similarly, the exotherm pertaining to crystallization of ice diminishes, whereas the step in heat flow associated with the glass transition becomes more pronounced in Fig. 3(a). At $x=0.13$ (blue line) the phenomenology changes and the exotherm corresponding to ice crystallization, and thus the no man's land, is virtually lost. Instead, another feature appears, namely, the exothermic first-order transition at $185 \mathrm{~K}$ that was attributed by Angell and collaborators to an LLPT. That is, the exotherm appears only in the solute-dominated region, which precludes that it can be assigned to an LLPT transition in water itself. By contrast to the exotherm associated with ice precipitation, this exotherm of unknown origin hardly shifts with changing mole fraction, and remains around $185 \mathrm{~K}$. As already mentioned by Zhao and Angell [12], it does, however, decrease in intensity at $x \geqslant 0.20$ and develops to a simple glass transition at $x=0.40$. Since the residual glass transition occurs at the same temperature as the liquid-to-glass transition of FCS, we conclude that the FCS also has a composition of $x \approx 0.40$. 
The final test to check for water polyamorphism is done using calorimetry on solutions after pressurization to $1.8 \mathrm{GPa}$ at $77 \mathrm{~K}$ [Fig. 3(b)]. For pure water, this procedure leads to HDA. HDA has a unique thermal signature at low temperatures [28], which is nicely reproduced in our scans [see black trace in Fig. 3(b)]: First, there is an exothermic transition signifying the polyamorphic HDA $\rightarrow$ LDA transition near $115 \mathrm{~K}$. Then LDA cold-crystallizes to ice I in another exotherm near 160 K. Similar traits are found for all solutions in the waterdominated region, i.e., at $x \leqslant 0.12$. However, the size of the exotherms diminishes with increasing $x$. Increasing the solute concentration also delays the polyamorphic transition to higher temperatures but shifts the cold-crystallization temperature of LDA to lower temperatures. These temperature shifts imply continuous stabilization of HDA-like states and continuous destabilization of LDA-like states upon adding solute, consistent with suggestions from theoretical and experimental work done on other solutions [9,10,26,29]. Above $180 \mathrm{~K}$, two other events are observed that are not present in pure water. The first corresponds to the weak glass-to-liquid transition at $185 \mathrm{~K}$ resulting from the devitrification of glassy FCS to supercooled FCS. The second indicates exothermic coldcrystallization of supercooled FCS between 220 and $240 \mathrm{~K}$.

Once the solute-dominated region at $x=0.13$ is entered [see blue trace in Fig. 3(b)], the thermal behavior again changes abruptly. That is, the polyamorphic HDA $\rightarrow$ LDA and the LDA $\rightarrow$ ice I transitions are no longer observed and are instead replaced by an endotherm at $190 \mathrm{~K}$. The endotherm of unknown origin is immediately followed by a weak exothermic cold-crystallization event leading to ice I near $200 \mathrm{~K}$, as also seen in the $\mathrm{x}$-ray diffraction data in Fig. 1. Ice crystallization enforces an increase of $\mathrm{N}_{2} \mathrm{H}_{5}{ }^{+} \mathrm{TFA}^{-}$ concentration in the remaining solution, which then coldcrystallizes to TFA $\cdot 4 \mathrm{H}_{2} \mathrm{O}$ at $220-240 \mathrm{~K}$ as indicated by the second exotherm in Fig. 3(b) and consistent with $\mathrm{x}$-ray diffraction scans of Fig. 1. We emphasize that the most important feature in the context of this work is the endotherm at $190 \mathrm{~K}$. Zhao and Angell [12] have assigned this to the reverse transition of the putative LLPT, i.e., a transformation of LDL to HDL water. However, our solutions here have been compressed well beyond the pressures necessary to yield high-density states. Still, we find the same endotherm as Zhao and Angell who studied the solutions only at ambient pressures [12]. This insensitivity to pressure as evidenced by DSC as well as x-ray diffraction data presented above clearly bolsters the view that polyamorphism of water does not play a role in aqueous $\mathrm{N}_{2} \mathrm{H}_{5}{ }^{+} \mathrm{TFA}^{-}$mixtures at $x \geqslant 0.13$. Only at $x \leqslant 0.12$, distinct features of water such as rapid crystallization of ice and HDA-LDA polyamorphism reappear. Alternatives to the ideal mixture hypothesis need to be sought in order to explain the extraordinary phase behavior of $\mathrm{N}_{2} \mathrm{H}_{5}{ }^{+} \mathrm{TFA}^{-}$solutions.

It remains to be answered what the origin of the exotherm upon cooling and endotherm upon heating is, if it is not related to an LLPT in pure water. We speculate that complex nanophase-separation processes in the solution cause these thermal features. Such processes have been proposed by Le and Molinero in simulations for electrolyte solutions [30] and by Lane et al. for eutectic solutions of $\mathrm{LiCl}$ and $\mathrm{LiSCN}$ [31]. This speculation is fueled by the halo peak observed at $14.5^{\circ}$, which is a typical diffraction angle for nanosized objects. Also our observation that cold-crystallization of FCS yields TFA $\cdot 4 \mathrm{H}_{2} \mathrm{O}$ and not crystals of $\mathrm{N}_{2} \mathrm{H}_{5}{ }^{+} \mathrm{TFA}^{-}$speaks in favor of separation processes in solution (see Bragg peaks marked with asterisks in Fig. 1 for $x=0.16$ ). Finally, the observation of double halos in liquid solutions (Fig. S2 in the Supplemental Material [15]), but triple halos in vitrified solutions (Fig. 1) is yet another hint to segregation processes in the solution on the nanoscale. Even though future work is necessary to investigate these speculations in more detail, clearly a liquid-liquid transition in water is not the driver for the transition at $185 \mathrm{~K}$.

Acknowledgments. We are grateful to the Austrian Science Fund FWF (Project No. I1392) for funding.

J.B. and L.-R.F. measured the data. J.B. and T.L. designed the study and wrote the manuscript.
[1] M. Chaplin, Water Structure and Science (London South Bank University, London, 2020), http://www1.lsbu.ac.uk/water/, accessed June, 2020.

[2] P. G. Debenedetti, J. Phys.: Condens. Matter 15, R1669 (2003); P. H. Handle, T. Loerting, and F. Sciortino, Proc. Natl. Acad. Sci. USA 114, 13336 (2017).

[3] P. H. Poole, F. Sciortino, U. Essmann, and H. E. Stanley, Nature (London) 360, 324 (1992).

[4] O. Mishima, L. D. Calvert, and E. Whalley, Nature (London) 314, 76 (1985).

[5] N. J. English and J. S. Tse, Phys. Rev. Lett. 106, 037801 (2011); A. K. Soper, J. Chem. Phys. 150, 234503 (2019); J. Teixeira, Substantia 3, 57 (2019).

[6] J. C. Palmer, P. H. Poole, F. Sciortino, and P. G. Debenedetti, Chem. Rev. 118, 9129 (2018).

[7] J. A. Sellberg, C. Huang, and A. Nilsson, Nature (London) 510, 381 (2014); K. H. Kim and F. Caupin, J. Non-Cryst. Solids 407, 441 (2015); C. Goy, M. A. C. Potenza, S. Dedera, M. Tomut, E. Guillerm, A. Kalinin, K. O. Voss, A. Schottelius,
N. Petridis, A. Prosvetov et al., Phys. Rev. Lett. 120, 015501 (2018).

[8] O. Mishima, J. Chem. Phys. 123, 154506 (2005); J. Bachler, P. H. Handle, N. Giovambattista, and T. Loerting, Phys. Chem. Chem. Phys. 21, 23238 (2019); C. A. Angell, Chem. Rev. 102, 2627 (2002).

[9] Y. Suzuki, J. Chem. Phys. 149, 204501 (2018).

[10] J. W. Biddle, V. Holten, and M. A. Anisimov, J. Chem. Phys. 141, 074504 (2014).

[11] C. A. Angell, Science 319, 582 (2008).

[12] Z. Zhao and C. A. Angell, Angew. Chem. 55, 2474 (2016).

[13] S. Woutersen, B. Ensing, M. Hilbers, Z. Zhao, and C. A. Angell, Science 359, 1127 (2018)

[14] R. Leberman and A. K. Soper, Nature (London) 378, 364 (1995).

[15] See Supplemental Material at http://link.aps.org/supplemental/ 10.1103/PhysRevE.102.060601 for detailed experimental procedures and methods as well as additional diffraction and calorimetry data, including Refs. [32-41]. 
[16] K. Winkel, M. Bauer, E. Mayer, M. Seidl, M. S. Elsaesser, and T. Loerting, J. Phys.: Condens. Matter 20, 494212 (2008).

[17] T. Loerting, K. Winkel, M. Seidl, M. Bauer, C. Mitterdorfer, P. H. Handle, C. G. Salzmann, E. Mayer, J. F. Finney, and D. T. Bowron, Phys. Chem. Chem. Phys. 13, 8783 (2011).

[18] K. Winkel, M. S. Elsaesser, E. Mayer, and T. Loerting, J. Chem. Phys. 128, 044510 (2008); C. M. Tonauer, M. Seidl-Nigsch, and T. Loerting, J. Phys.: Condens. Matter 30, 034002 (2018).

[19] O. Mishima, J. Chem. Phys. 100, 5910 (1994).

[20] I. Kohl, E. Mayer, and A. Hallbrucker, Phys. Chem. Chem. Phys. 3, 602 (2001).

[21] O. Mishima, L. D. Calvert, and E. Whalley, Nature (London) 310, 393 (1984).

[22] G. N. Ruiz, L. E. Bove, H. R. Corti, and T. Loerting, Phys. Chem. Chem. Phys. 16, 18553 (2014).

[23] Y. Suzuki and O. Mishima, J. Chem. Phys. 138, 084507 (2013).

[24] I. Popov, A. Greenbaum (Gutina), A. P. Sokolov, and Y. Feldman, Phys. Chem. Chem. Phys. 17, 18063 (2015).

[25] A. Inaba and O. Andersson, Thermochim. Acta 461, 44 (2007).

[26] J. Bachler, V. Fuentes-Landete, D. A. Jahn, J. Wong, N. Giovambattista, and T. Loerting, Phys. Chem. Chem. Phys. 18, 11058 (2016).

[27] J. E. K. Schawe, Thermochim. Acta 451, 115 (2006).

[28] Y. P. Handa, O. Mishima, and E. Whalley, J. Chem. Phys. 84, 2766 (1986).

[29] D. A. Jahn, J. Wong, J. Bachler, T. Loerting, and N. Giovambattista, Phys. Chem. Chem. Phys. 18, 11042 (2016).
[30] L. Le and V. Molinero, J. Phys. Chem. A 115, 5900 (2011).

[31] P. D. Lane, J. Reichenbach, A. J. Farrell, L. A. I. Ramakers, K. Adamczyk, N. T. Hunt, and K. Wynne, Phys. Chem. Chem. Phys. 22, 9438 (2020).

[32] S. Klotz, T. Strässle, A. M. Saitta, G. Rousse, G. Hamel, R. J. Nelmes, J. S. Loveday, and M. Guthrie, J. Phys.: Condens. Matter 17, S967 (2005).

[33] M. Seidl, K. Amann-Winkel, P. H. Handle, G. Zifferer, and T. Loerting, Phys. Rev. B 88, 174105 (2013).

[34] J. N. Stern, M. Seidl-Nigsch, and T. Loerting, Proc. Natl. Acad. Sci. USA 116, 9191 (2019).

[35] K. Amann-Winkel, C. Gainaru, P. H. Handle, M. Seidl, H. Nelson, R. Böhmer, and T. Loerting, Proc. Natl. Acad. Sci. USA 110, 17720 (2013); V. Fuentes-Landete, L. J. Plaga, M. Keppler, R. Böhmer, and T. Loerting, Phys. Rev. X 9, 011015 (2019).

[36] A. Bogdan, M. J. Molina, H. Tenhu, and T. Loerting, J. Phys. Chem. A 119, 4515 (2015); A. Hauptmann, K. F. Handle, P. Baloh, H. Grothe, and T. Loerting, J. Chem. Phys. 145, 211923 (2016).

[37] A. Baudot and V. Odagescu, Cryobiology 48, 283 (2004).

[38] P. Gallo, K. Amann-Winkel, C. A. Angell, M. A. Anisimov, F. Caupin, C. Chakravarty, E. Lascaris, T. Loerting, A. Z. Panagiotopoulos, J. Russo et al., Chem. Rev. 116, 7463 (2016).

[39] T. S. Zwier, Science 304, 1119 (2004).

[40] D. Mootz and D. Boenigk, Z. Naturforschung B 39, 298 (1984).

[41] Y. Suzuki and O. Mishima, J. Phys.: Condens. Matter 21, 155105 (2009). 